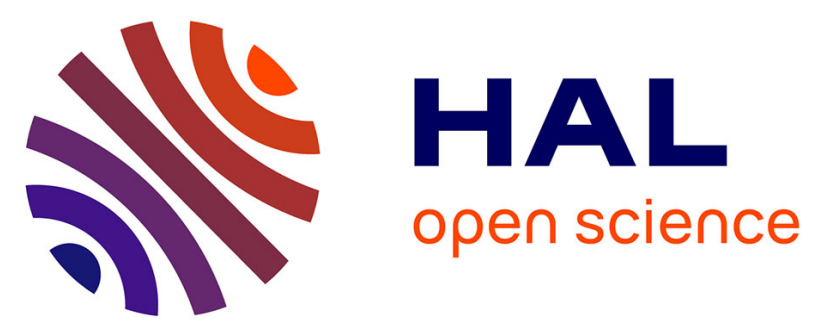

\title{
Picosecond synchronously pumped optical parametric oscillator based on chirped quasi-phase matching
}

Guillaume Walter, Delphine Descloux, Jean-Baptiste Dherbecourt, Jean-Michel Melkonian, Myriam Raybaut, Cyril Drag, Antoine Godard

\section{- To cite this version:}

Guillaume Walter, Delphine Descloux, Jean-Baptiste Dherbecourt, Jean-Michel Melkonian, Myriam Raybaut, et al.. Picosecond synchronously pumped optical parametric oscillator based on chirped quasi-phase matching. Journal of the Optical Society of America B, 2020, 37 (2), pp.552-563. 10.1364/JOSAB.380605 . hal-02470302

\section{HAL Id: hal-02470302 \\ https://hal.science/hal-02470302}

Submitted on 7 Feb 2020

HAL is a multi-disciplinary open access archive for the deposit and dissemination of scientific research documents, whether they are published or not. The documents may come from teaching and research institutions in France or abroad, or from public or private research centers.
L'archive ouverte pluridisciplinaire HAL, est destinée au dépôt et à la diffusion de documents scientifiques de niveau recherche, publiés ou non, émanant des établissements d'enseignement et de recherche français ou étrangers, des laboratoires publics ou privés. 


\title{
Picosecond synchronously-pumped optical parametric oscillator based on chirped quasi-phase matching
}

\author{
Guillaume Walter $^{1}$, Delphine Descloux ${ }^{1}$, Jean-Baptiste Dherbecourt ${ }^{1}$, Jean-Michel \\ Melkonian $^{1}$, Myriam Raybaut ${ }^{1}$, Cyril Drag ${ }^{2}$, and Antoine Godard ${ }^{1, *}$ \\ ${ }^{1}$ DPHY, ONERA, Université Paris Saclay, 91123 Palaiseau cedex, France \\ ${ }^{2}$ Laboratoire de Physique des Plasmas (UMR 7648), CNRS, Univ. Paris-Saclay, Sorbonne Université, Ecole polytechnique, 91128 Palaiseau, France \\ *Corresponding author: antoine.godard@onera.fr \\ Compiled December 24, 2019
}

\begin{abstract}
We investigate and model a picosecond synchronously pumped optical parametric oscillator (OPO) based on an aperiodically poled lithium niobate (APPLN) nonlinear crystal with a chirped quasi-phasematching (QPM) grating. We observe remarkable spectral features with an asymmetric OPO spectrum consisting of a main peak with lower side-lobes. Depending on the sign of the QPM chirp rate, the sidelobes are located either on the red or on the blue side of the main peak. Meanwhile, side-bands develop in the depleted pump spectrum. We attribute these features to cascaded sum-/difference-frequency generation processes which are quasi-phase matched at different positions in the APPLN crystal. A terahertzgeneration cascading effect is also observed and characterized at high pump power. ๑ 2019 Optical Society of America
\end{abstract}

http://dx.doi.org/10.1364/ao.XX.XXXXXX

\section{INTRODUCTION}

Aperiodic or chirped quasi-phase-matching (QPM) enables the engineering of the optical frequency conversion processes in a nonlinear material [1]. Used as the amplification medium in an optical parametric oscillator (OPO), a chirped QPM crystal can provide a broad parametric gain spectrum without the need to use short crystals with reduced conversion efficiency or to match the group velocities of the interacting waves [2], which is only possible for particular wavelengths for a given nonlinear material and often requires noncollinear phase matching geometries, limiting the interaction length for focused beams.

Lithium niobate is a versatile nonlinear material for the practical implementation of aperiodic QPM owing to the capability of creating a modulation of its nonlinear susceptibility by ferroelectric domain electric-field poling [3]. Such microstructured bulk lithium niobate crystals are called aperiodically poled lithium niobate (APPLN). APPLN crystals allow a large number of applications in various spectral and temporal domains. In the femtosecond regime, they are especially used for pulse amplification and shaping $[4,5]$, but also for high conversion efficiency OPOs [6]. APPLN has been used in the nanosecond regime, with a reported OPO with remarkable spectral properties in transient and steady-state regime [7]. One can also mention the use of APPLN waveguides for frequency comb upconversion by sum-frequency generation (SFG) with a continuous-wave laser [8]. In our previous studies, we exploited the broad parametric gain bandwidth provided by chirped APPLN, in com- bination with intracavity spectral filter, to demontrate rapidly tunable synchronouly-pumped OPOs (SPOPOs) in the picosecond regime $[9,10]$. We also studied an APPLN-based broadband picosecond SPOPO without intracavity filter and reported particular spectrotemporal dynamics [11, 12].

Here, we present a detailed study of a similar broadband picosecond SPOPO based on a chirped QPM crystal. In particular, we thoroughly investigate its remarkable spectral features in steady-state operation that were overlooked in our previous paper, i.e. the emission of an asymmetric OPO spectrum consisting of a main peak with lower side-lobes which are located either on the red or on the blue side of the main peak, depending on the sign of the QPM chirp rate. Experimental and numerical investigations lead us to attribute these features to cascaded sum-/difference-frequency generation (SFG/DFG) processes which are quasi-phase matched at different positions in the APPLN crystal. In addition, we also report on a terahertz-wave generation cascading effect which occurs at high pump power.

This paper is organized as follows. Section 2 is devoted to the design and the characterization of the parametric gain spectrum of the APPLN crystal. The OPO experimental setup is then described in Section 3. Section 4 presents the investigation of the OPO efficiency as a function of the QPM chirp rate and its sign. Section 5, which is the core of this paper, is devoted to the experimental and numerical investigation of the steady-state spectral properties of the SPOPO and the related discussion. Finally, the spectral properties of the OPO at high pump power 
are studied in Section 6, where a terahertz cascading effect is observed. The final section summarizes the conclusions.

\section{DESIGN AND CHARACTERIZATION OF THE APPLN CRYSTALS}

\section{A. Design}

We designed a 60-mm-long 5\%-MgO-doped APPLN crystal containing multiple gratings with different QPM chirp rates in order to investigate different gain bandwidths. The central grating period, $\Lambda_{0}=29.15 \mu \mathrm{m}$, is the same for all the gratings, and corresponds to the perfect phase matching point period of a $1470 \mathrm{~nm}$ signal parametric generation from a $1064 \mathrm{~nm}$ pump.The QPM chirp rate, $\kappa^{\prime}$, is defined as

$$
\kappa^{\prime}=\left.\frac{\mathrm{d} K_{\mathrm{g}}(z)}{\mathrm{d} z}\right|_{z=L_{\mathrm{c}} / 2} .
$$

where $K_{\mathrm{g}}(z)=2 \pi / \Lambda(z)$ is the grating wavevector with $z$ the axial direction and $z=L_{\mathrm{c}} / 2$ the grating center, with $L_{\mathrm{c}}$ the grating length.

The smallest chirp rate was chosen to yield the same gain with the $60-\mathrm{mm}$ APPLN crystal (for the wavelength which is quasi-phase-matched in the grating center) as a 20 -mm uniform periodically poled lithium niobate (PPLN) crystal, i.e. with $\kappa^{\prime}=0$. The other QPM gratings were designed with a gradual increase of the gain bandwidth from one grating to the other. We chose a 20-mm uniform PPLN crystal as a reference, since we previously carried out extensive picosecond OPO experiments with such a crystal [13].

The APPLN crystal QPM grating profile was designed following the approaches developed by Charbonneau-Lefort et al. in [1]. In a first step, we used the convenient gain-bandwidth product formulas (41) and (42) of [1], to carry out a preliminary design of the chirped QPM gratings in comparison with the uniform QPM grating of the 20-mm crystal. According to these formulas, derived in large-gain regime in the undepleted-pump approximation, one can approximately relate the logarithmicgain-bandwidth products of chirped and uniform QPM gratings as follows:

$$
\ln G_{\mathrm{C}} \times \Delta \omega_{\mathrm{c}} / L_{\mathrm{c}}=\frac{\pi}{4} \ln G_{\mathrm{u}} \times \Delta \omega_{\mathrm{u}} / L_{\mathrm{u}}
$$

where $G_{m}$ is the gain of the QPM grating, $\Delta \omega_{m}$ is the FWHM amplification bandwidth, and $L_{m}$ is the length of the QPM grating, while subscript $m=\mathrm{c}$ refers to the chirped QPM grating and $m=\mathrm{u}$ to the uniform QPM grating. Moreover, for the chirped QPM grating, it can be shown that:

$$
\Delta \omega_{\mathrm{c}}=\left|\kappa^{\prime} \delta v_{\mathrm{si}}\right| L_{\mathrm{c}}
$$

where $\delta v_{\mathrm{si}}$ is the group-velocity mismatch parameter defined as:

$$
\frac{1}{\delta v_{\mathrm{si}}}=\frac{1}{v_{\mathrm{s}}}-\frac{1}{v_{\mathrm{i}}}
$$

with $v_{\mathrm{s}}$ the group velocity of the signal and $v_{\mathrm{i}}$ the group velocity of the idler.

Considering the two crystal lengths $\left(L_{\mathrm{u}}=20 \mathrm{~mm}\right.$ and $L_{\mathrm{c}}=60 \mathrm{~mm}$ ), we can thus expect to achieve $G_{\mathrm{c}} \simeq G_{\mathrm{u}}$ with $\Delta \omega_{\mathrm{c}} \simeq 3 \Delta \omega_{\mathrm{u}}$. In the case of the 20-mm uniform QPM PPLN crystal, the bandwidth is measured to be $\Delta \lambda_{\mathrm{u}}=2.3 \mathrm{~nm}$. The corresponding chirp rate that gives the same parametric gain in the $60 \mathrm{~mm}$ long APPLN crystal is thus roughly $\kappa_{\text {ref }}^{\prime}=1.6 \times 10^{4} \mathrm{~m}^{-2}$
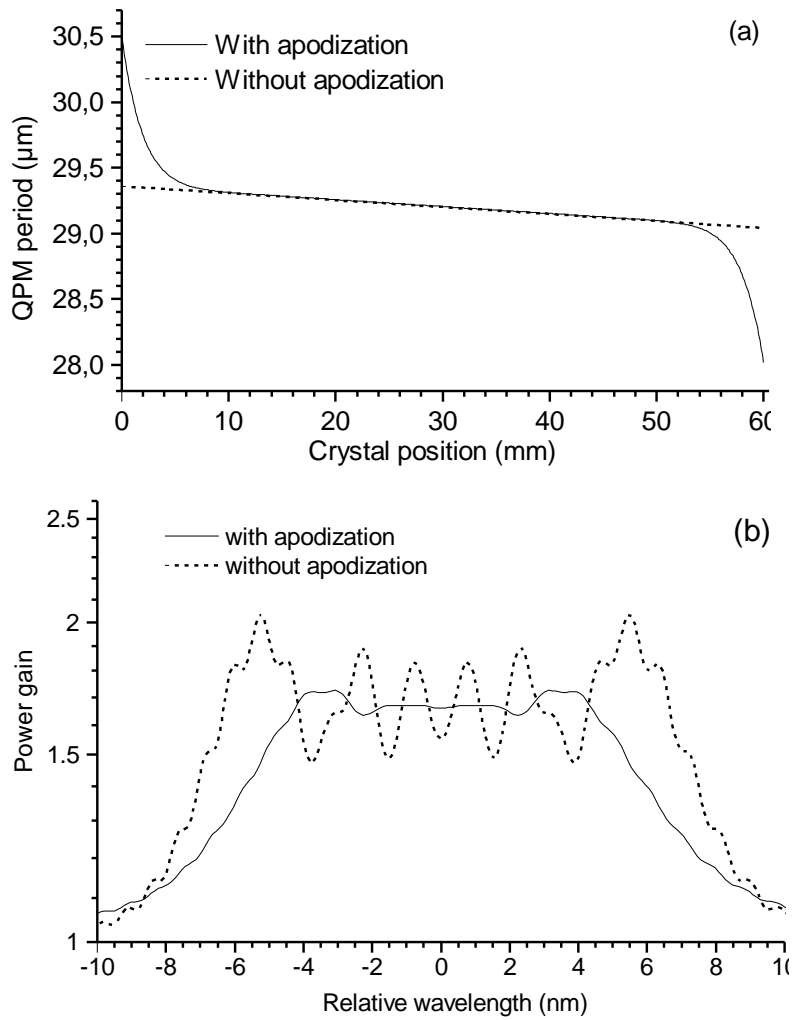

Fig. 1. (a) QPM period as a function of the distance from the crystal center, for grating G4, for the parameters listed in table 1. (b) Corresponding parametric gain spectrum with reduction of the ripples owing to apodization. For both figures, the dash curve corresponds to a grating without apodization and with the same QPM chirp rate as grating $\mathrm{G} 4\left(\mu_{1}=\mu_{2}=0\right)$.

at the considered wavelengths of $1470 \mathrm{~nm}$, where $\delta v_{\text {si }}$ was calculated by use of the $5 \%$-MgO-doped $\mathrm{LiNbO}_{3}$ Sellmeier equation provided in [14]. For larger chirp values $\kappa^{\prime}>\kappa_{\text {ref }}^{\prime}$, the gain is expected to decrease at the inverse power of the ratio of the chirp rates: $\kappa_{\text {ref }}^{\prime} / \kappa^{\prime}$. We then carried out modeling to refined the design with these engineering rules in mind.

\section{B. Modeling}

Refined design of the aperiodic gratings was carried out with calculations in the picosecond regime. For that purpose, we used a homemade plane-wave simulation code based on a finite difference method in temporal domain to solve the coupled nonlinear equations, taking into account group velocity mismatch and dispersion using a similar set of equations as in [15].

In order to take the finite beam transverse profiles into account, we applied a correction factor $C=0.25$ to the nonlinear coupling parameter $d_{\text {eff }}$ used in the plane-wave calculation, i.e. $C d_{\text {eff }}=4 \mathrm{pm} / \mathrm{V}$ instead of $16 \mathrm{pm} / \mathrm{V}$. This correction factor was estimated experimentally from an adaptation of the FindlayClay method to OPOs [16], which was applied to our SPOPO based on a $20 \mathrm{~mm}$ uniform PPLN crystal. This method basically consists in measuring the SPOPO oscillation threshold for several output couplers with different reflectivities. One can then deduce the cavity losses and correction factor $C$ as bestfit parameters to match experiment with equation (12) of [16]. The same value of correction factor $C$ can then be used for OPA 
calculation since pump and signal beam sizes and focusing are similar as those involved in the SPOPO.

The spectral ripples in the parametric gain spectra were reduced following the approach developed in [1] with the aperiodic QPM gratings consisting of a linearly chirped central section between two nonlinearly chirped sections for apodization, as presented in Fig. 1(a).

For that purpose, we selected QPM grating profiles with a grating wavevector expressed as:

$$
\begin{aligned}
K_{\mathrm{g}}(z)= & \frac{2 \pi}{\Lambda_{0}}+\kappa^{\prime}\left(z-\frac{L_{\mathrm{c}}}{2}\right) \\
& +\mu_{1} \frac{\kappa^{\prime} L_{\mathrm{c}}}{2}\left(\frac{2 z}{L_{\mathrm{c}}}-1\right)^{\nu_{1}}+\mu_{2} \frac{\kappa^{\prime} L_{\mathrm{c}}}{2}\left(\frac{2 z}{L_{\mathrm{c}}}-1\right)^{v_{2}},
\end{aligned}
$$

where the two last terms are added to the linear chirp profile to provide apodization. They are both large, odd powers where $\mu_{1,2}$ are amplitudes of the departure from linearity and $v_{1,2}$ are large, odd integers. The grating period $\Lambda_{\mathrm{g}}(z)$ can then be straightforwardly determined with $\Lambda_{\mathrm{g}}(z)=2 \pi / K_{\mathrm{g}}(z)$. As seen in Fig. 1(b), the spectral ripples of the parametric gain curve are efficiently suppressed owing to apodization sections. This approach led to the design of a crystal containing seven aperiodic QPM gratings whose parameters are listed in table 1.

Table 1. Parameters used for chirped QPM gratings of the APPLN crystal and corresponding calculated parametric gain bandwidths at the signal and idler wavelengths (FWHM). For all gratings the central QPM period is $\Lambda_{0}=29.15 \mu \mathrm{m}$.

\begin{tabular}{llllllll}
\hline Grating & $\kappa^{\prime}$ & $\mu_{1}$ & $v_{1}$ & $\mu_{2}$ & $v_{2}$ & $\begin{array}{l}\Delta \lambda_{s} \\
(\mathrm{~nm})\end{array}$ & $\begin{array}{l}\Delta \lambda_{i} \\
(\mathrm{~nm})\end{array}$ \\
\hline G1 & $1.8 \times 10^{4}$ & 2.6 & 21 & 0 & 0 & 7 & 48.1 \\
G2 & $2.8 \times 10^{4}$ & 8.03 & 15 & 0 & 0 & 8.3 & 57 \\
G3 & $3.2 \times 10^{4}$ & 1.48 & 21 & 0 & 0 & 11.7 & 80 \\
G4 & $3.9 \times 10^{4}$ & 6.8 & 15 & 0 & 0 & 13.4 & 92 \\
G4' & $3.9 \times 10^{4}$ & 4.2 & 9 & 4.6 & 39 & 13.4 & 92 \\
G5 & $4.75 \times 10^{4}$ & 1.9 & 39 & 0 & 0 & 17.8 & 122 \\
G6' & $1.17 \times 10^{5}$ & 7.8 & 17 & 6.8 & 39 & 40 & 275 \\
\hline
\end{tabular}

\section{OPA experimental characterization}

Two AR-coated 60-mm APPLN crystals (provided by HC Photonics) were supplied according to the grating parameters of table 1. The first crystal contains gratings G1, G2, G3, G4, and G5. The second crystal also contains gratings G4' and G6'. To assess our design, we implemented an optical parametric amplifier (OPA) experiment based on the first APPLN crystal in order to measure the parametric curve of the QPM gratings.

The OPA setup is shown in Fig. 2. The output beam from a 1064-nm, picosecond, nearly Fourier-transform limited, modelocked pump laser (PicoTRAIN, High Q Laser), with a $76 \mathrm{MHz}$ pulse repetition rate, a 8 ps pulse duration (provided by supplier), and a $<0.3 \mathrm{~nm}$ spectral bandwidth (measured), is sent on a polarizing cube beamsplitter preceded by a half-wave plate so as to split it in two parts with an adjustable power ratio. The first one, containing typically $60 \%$ of the $17-\mathrm{W}$ incident power, was sent to a delay line and then focused in the APPLN crystal

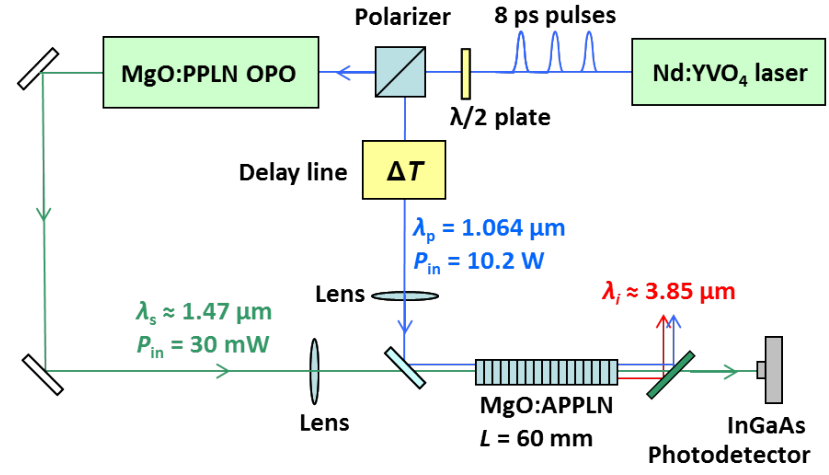

Fig. 2. Schematic diagram of the OPA experimental setup.

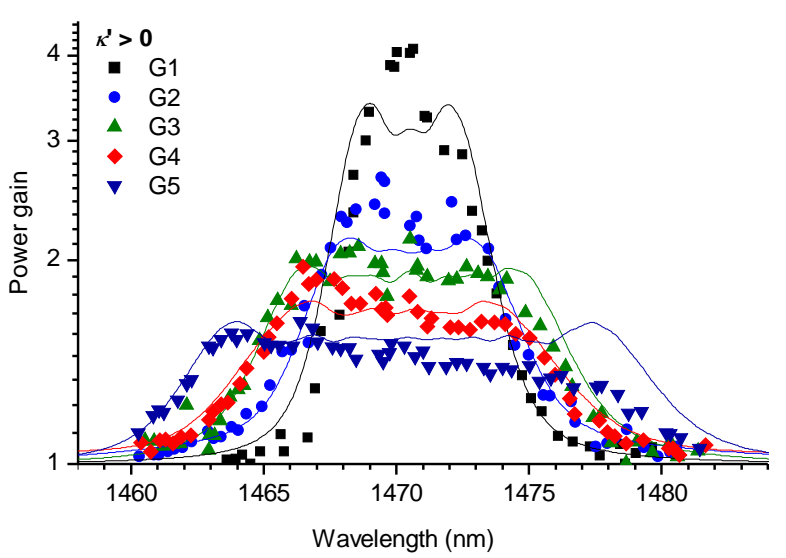

Fig. 3. Parametric gain spectrum for QPM gratings G1-G5 (Solid lines: numerical calculations; symbols: experimental measurements).

to pump the OPA. The remaining of the pump power was used to pump a SPOPO based on the 20-mm PPLN crystal and containing an intracavity Littrow-mounted diffraction grating. Such a scheme enables to carry out fine tuning of the signal wavelength and to deliver nearly Fourier-transform limited pulses $(\mathrm{FWHM} \simeq 0.5 \mathrm{~nm})$. The properties of this SPOPO were already detailed in [13].

The pump and signal waist radiuses were both set to $150 \mu \mathrm{m}$, to have both Rayleigh lengths in the same range as the nonlinear crystal length. For each wavelength, the delay between the incident pump and signal pulses was adjusted to maximize the OPA gain.

As shown in Fig. 3, we obtained a good agreement between measured and calculated gain curves in the small-signal model. As expected from theory, the product of the logarithmic gain by the bandwidth is almost constant, which confirms the relevance of the design formulas derived in [1]. One can nevertheless notice a slight asymmetry in the experimental gain spectra. This feature is attributed to imperfect focusing of the input beam that leads to high intensities close to the output end of the crystal. A mirror image of the spectra was indeed obtained by flipping the orientation of the APPLN crystal.

\section{OPO EXPERIMENTAL SETUP}

Our SPOPO is based on the 60-mm long MgO-doped APPLN crystal, pumped by the same $1.064-\mu \mathrm{m}$ mode-locked picosecond 


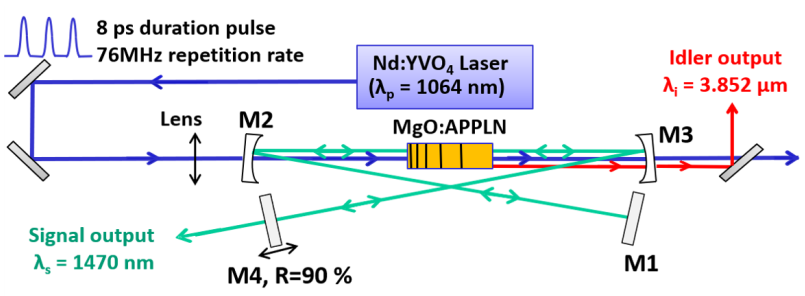

Fig. 4. Experimental setup of the picosecond SPOPO based on the chirped QPM APPLN crystal.

laser. As shown in Fig. 4, the SPOPO resonator has a standingwave configuration with a central focused arm between two collimated arms. This oscillator is singly resonant at the signal wavelength around $1.47 \mu \mathrm{m}$ while the idler beam at $3.85 \mu \mathrm{m}$ is totally transmitted through the mirror after the crystal exit facet. The radius of curvature of both spherical mirrors, M2 and M3, is $500 \mathrm{~mm}$, resulting in an estimated signal waist radius at $1 / e^{2}$ of $150 \mu \mathrm{m}$. Mirrors M1, M2, and M3 are highly reflective at the signal wavelength. The plane output coupler M4 is mounted on a translation stage so that the cavity length can be slightly changed to optimize the synchronous-pumping condition. In most of the reported experiments, M4 has a reflectivity $R_{\mathrm{Oc}}=$ $90 \%$ at the signal wavelength. A coupler with a reflectivity of $98 \%$ can also we used to reduce the oscillation threshold for grating G6' with the highest chirp rate. The pump beam is focused in the middle of the crystal. For first experiments, its waist radius was set to $150 \mu \mathrm{m}$ as in [13]. Then, we reduced it to $110 \mu \mathrm{m}$ so as to reach the same parametric gain with a lower current of pump diodes of the 1.064- $\mu \mathrm{m}$ laser. To change the sign, positive or negative, of the QPM chirp rate, we can flip the orientation of the APPLN crystal in the cavity.

The signal pulses were characterized by use of an interferometric autocorrelator based on two-photon absorption in a silicon photodetector (Thorlabs, Det10 A) at the output of a Michelson interferometer. We observed the signal beam profile by use of a beam profiling camera (Pyrocam, Ophir), which possesses an active matrix of $124 \times 124$ pixels of $100 \times 100 \mu \mathrm{m}^{2}$. Spectral characterizations were carried out with an optical spectrum analyzer (OSA, Yokogawa) with a wavelength resolution of $10 \mathrm{pm}$.

\section{OPO INPUT-OUTPUT CHARACTERISTICS}

\section{A. Experiment}

For each QPM grating, we measure an oscillation threshold power that does not depend on the crystal orientation. Fig. 5 shows the corresponding value of the threshold average power for each QPM grating. Excluding grating G5, we observe a linear variation of the threshold power with the chirp rate magnitude. Such a linear variation is consistent with the expected dependence of the parametric gain with the chirp rate. Indeed, according to equation (17) of [1], the small-signal peak parametric gain can be approximated by

$$
\Gamma=\exp \left(2 \pi \gamma^{2} / \kappa^{\prime}\right)
$$

where $\Gamma$ is the power gain experienced by the signal for each pass in the APPLN crystal and $\gamma$ is the coupling coefficient defined as

$$
\gamma^{2}=\frac{\omega_{\mathrm{s}} \omega_{\mathrm{i}}}{n_{\mathrm{s}} n_{\mathrm{i}} n_{\mathrm{p}}} \frac{2 d_{\mathrm{eff}}^{2}}{\epsilon_{0} c^{3}} I_{\mathrm{p}},
$$

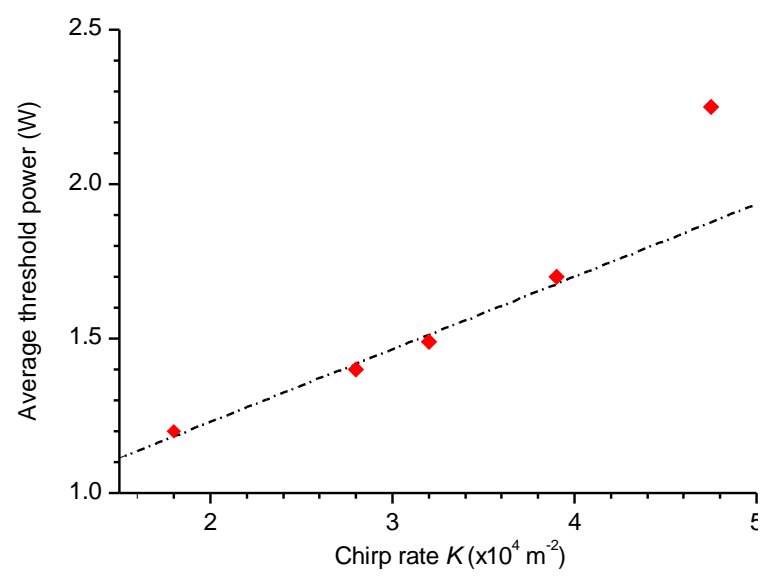

Fig. 5. OPO average power threshold as a function of the QPM chirp rate. The dashed-dotted line is a linear fit calculated without the highest chirp rate data point. The pump beam waist radius is $150 \mu \mathrm{m}$ and $R_{\mathrm{OC}}=90 \%$.

with $I_{\mathrm{p}}$ the pump intensity (in $\mathrm{W} / \mathrm{m}^{2}$ ), $\omega_{\mathrm{s}}$ and $\omega_{\mathrm{i}}$ the signal and idler angular frequencies, $n_{\mathrm{s}}, n_{\mathrm{i}}$ and $n_{\mathrm{p}}$ the signal, idler and pump refractive indices, $d_{\text {eff }}$ the effective nonlinear coefficient, $\epsilon_{0}$ the vacuum permittivity, and $c$ the light velocity. For constant cavity losses, the threshold intensity is thus proportional to $\kappa^{\prime}$ so as to maintain a constant gain. The discrepancy observed with grating G5 is unexpected since its measured gain spectrum was consistent with theoretical expectation. We currently do not have any explanation of this phenomenon since we did not observe any damage of the crystal.

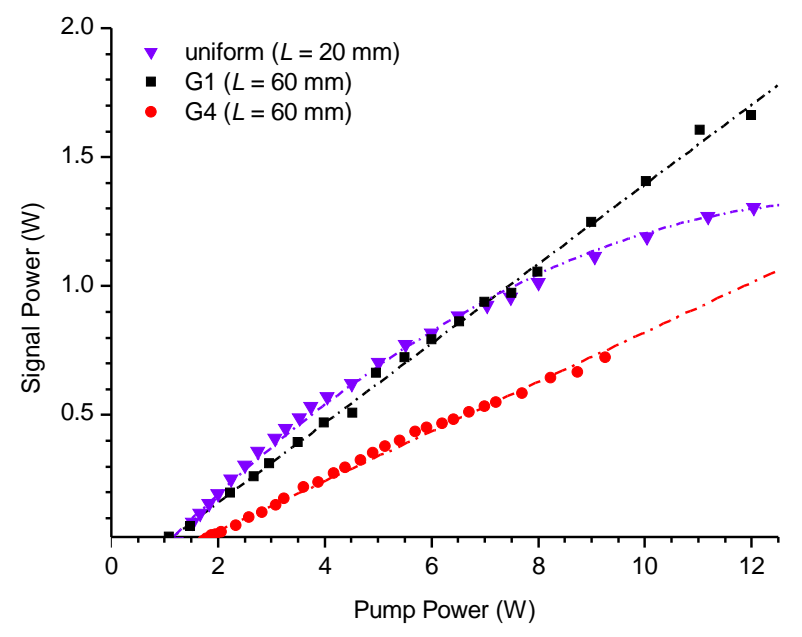

Fig. 6. Signal output power as a function of pump power for the SPOPO based on the 20-mm-long PPLN crystal and on gratings G1 and G4 of the 60-mm-long APPLN crystal. Symbols are experimental data points and lines are respectively a linear fit for QPM gratings G1 and G4, and a parabolic fit for the uniform QPM grating. The pump beam waist radius is $150 \mu \mathrm{m}$ and $R_{\mathrm{oc}}=90 \%$.

Fig. 6 shows the signal output power as a function of the input pump power for gratings G1 and G4, and for a similar SPOPO cavity containing the $20-\mathrm{mm}$ MgO:PPLN crystal with 


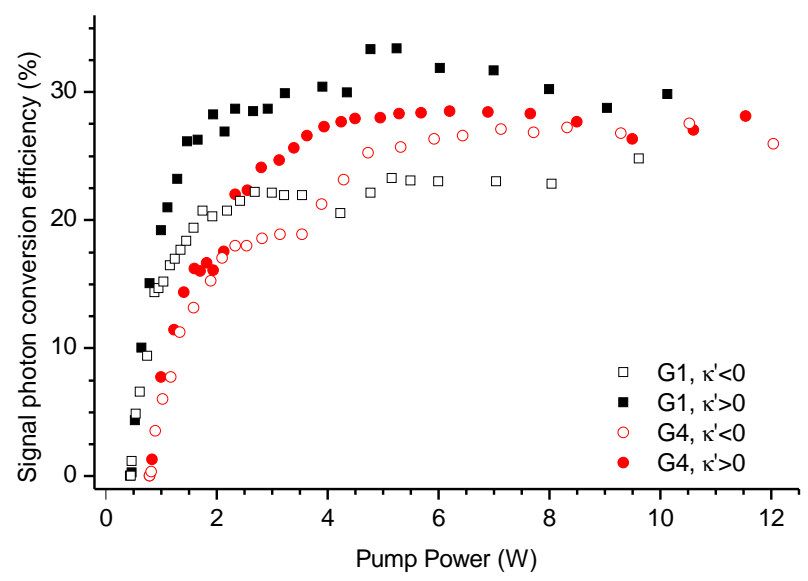

Fig. 7. Experimental signal photon conversion efficiency as a function of pump power for gratings G1 and G4 for both QPM chirp rate signs. The pump beam waist radius is $110 \mu \mathrm{m}$ and $R_{\mathrm{oc}}=90 \%$.

a uniform QPM period. As expected from the intended design, the oscillation threshold is very similar for OPOs based either on grating G1 or on the 20-mm grating with a uniform QPM period. We can also notice that the use of an APPLN crystal leads to less saturation at high pump energy, which is consistent with the theorical prediction [17]. In our configuration, the quantum conversion efficiency remains nevertheless smaller than $20 \%$, meaning that the condition for adiabatic frequency conversion is not met. Indeed, neither the chirp rate nor the apodization profile were designed here for such a purpose $[18,19]$.

In order to improve the OPO efficiency, we then reduced the pump beam radius from $150 \mu \mathrm{m}$ to $110 \mu \mathrm{m}$. Additional characterizations were then carried out with such a higher focusing of the pump beam. Fig. 7 presents the corresponding results for gratings $\mathrm{G} 1$ and $\mathrm{G} 4$ for the two chirp rate signs.

This new maximum quantum conversion efficiency of $33.5 \%$, is obtained with grating G1 that has a lower chirp rate than grating G4. Below $10 \mathrm{~W}$ of pump power, we notice a difference between negative and positive chirp rates, with a conversion efficiency which seems to to favor positive chirp rates. Then, at higher pump power, we observe a higher saturation for this positive chirp rate leading eventually to very similar conversion efficiencies of about $28 \%$ for both chirp signs and both gratings, corresponding to a power slope efficiency of about $20 \%$ for the signal wave.

\section{B. Numerical simulation}

We carried out numerical simulation of the conversion efficiency of the OPO with our code. As shown in Fig. 8, the calculated input-output curves present a good qualitative agreement with the experimental results of Fig. 7. The calculated conversion efficiencies are of the same order as the measured ones and a similar difference is observed between positive and negative chirp rates. Our modeling code is thus able to reproduce the main observed features. To avoid overestimating back-conversion with the plane-wave code, we assume a round-trip loss of $10 \%$ and a correction factor $C=0.31\left(C d_{\text {eff }}=4.96 \mathrm{pm} / \mathrm{V}\right)$ which enables us to have a consistent conversion efficiency for the various chirp rates. This results in calculated oscillation thresholds higher than the experimental ones. This trade-off between a consistent conversion efficiency and an overestimated threshold has been

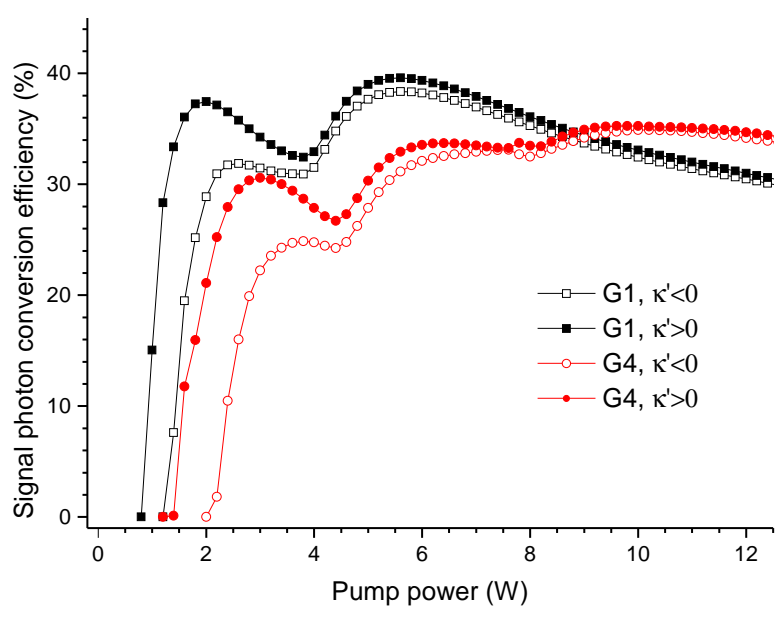

Fig. 8. Calculated signal photon conversion efficiency as a function of pump power for gratings G1 and G4 for both QPM chirp rate signs.

made, because the following of our study focuses on the investigation of the OPO features at steady-state for pumping rates well above the oscillation threshold. We thus selected numerical calculation parameters leading to realistic pump depletion to make numerical simulations a valid tool to analyze the steady-state spectral properties of the OPO.

\section{STEADY-STATE SPECTRUM}

\section{A. Experimental results}

For each investigated configuration, we measured the average spectrum, the spatial beam profile, and the temporal autocorrelation for several pumping rates. Fig. 9 presents the results for negative chirp rates while Fig. 10 corresponds to positive chirp rates. We systematically observe an asymmetric shape with a main peak and lower side-lobes. Depending on the chirp rate signs the side-lobes are located either on the red or the blue side of the main peak. Similar features are observed for both pump focusing configurations. The higher the pump intensity, the broader the emitted spectrum with more side-lobes. The lobes structures appear more regular in the case of a negative chirp rate. In both cases, the spatial beam profile of the signal remains nearly Gaussian in both near and far fields.

We also observe that the number of lobes and the spectral separation between them increase when the chirp rate is higher (not shown here), which leads to a wider total signal bandwidth.

The autocorrelation traces of the signal pulses broadens when the pump power increases. In contrast to the pump pulses which are nearly Fourier-transform-limited, the time-bandwidth product of the signal pulses typically spans from 2 to 10 times above the transform limit when pump power increases from the oscillation threshold to 5 times above threshold. In Figs. 10(g) and 10(h), one can notice a modulation of the envelope of the autocorrelation traces whose period of about 1.2 ps corresponds to the beating between two spectral lines with a $6 \mathrm{~nm}$ spacing (i.e almost $1 \mathrm{THz}$ at $1470 \mathrm{~nm}$ ), which is consistent with the spacing of the two main lobes of the signal spectra.

As shown in Fig. 11, we also measured the pump spectrum after passing through the chirped QPM crystal. When the OPO is oscillating, the pump also experiences a spectral shaping with side-lobes which develop at both sides of the main spectral 

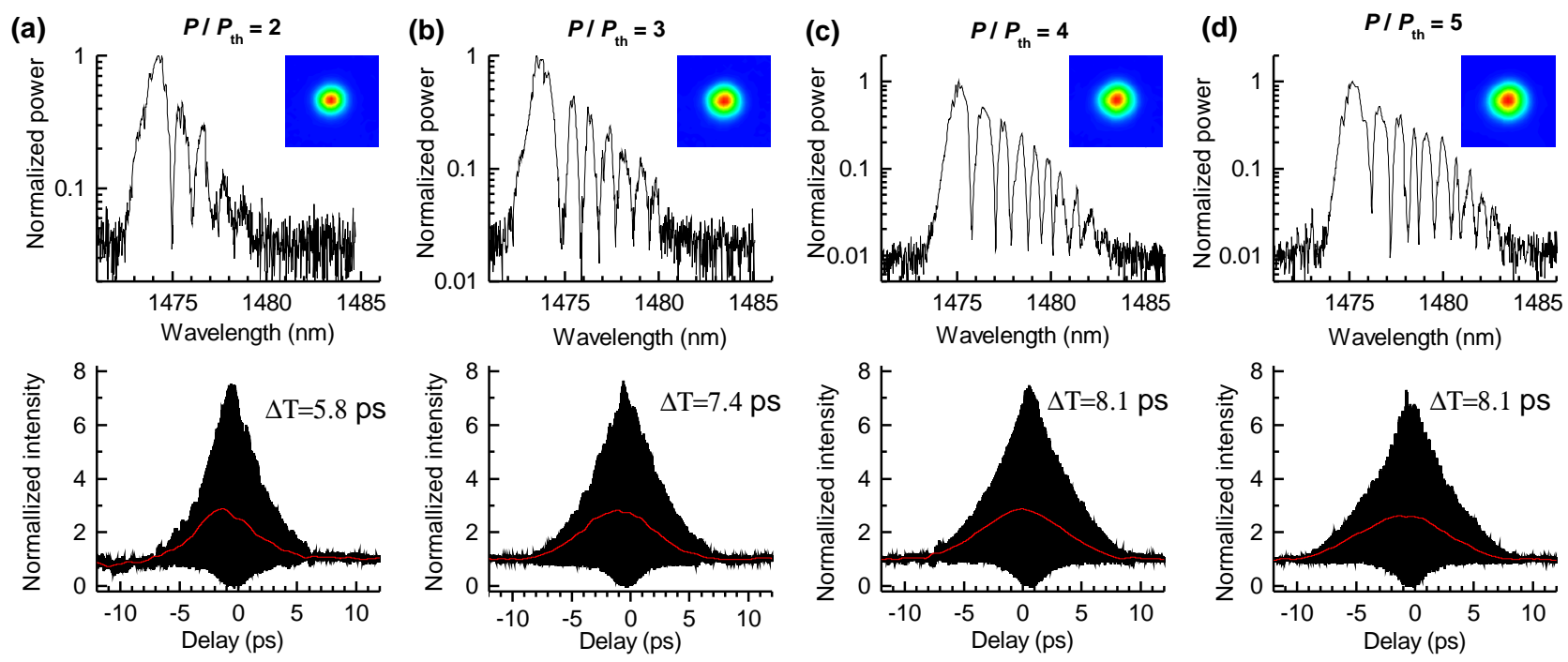

Fig. 9. Experimental signal spectrum, beam profile and autocorrelation trace for several pumping rates for QPM grating G4 with a negative QPM chirp rate, a $150 \mu m$ pump waist, and $R_{\mathrm{oc}}=90 \%$.
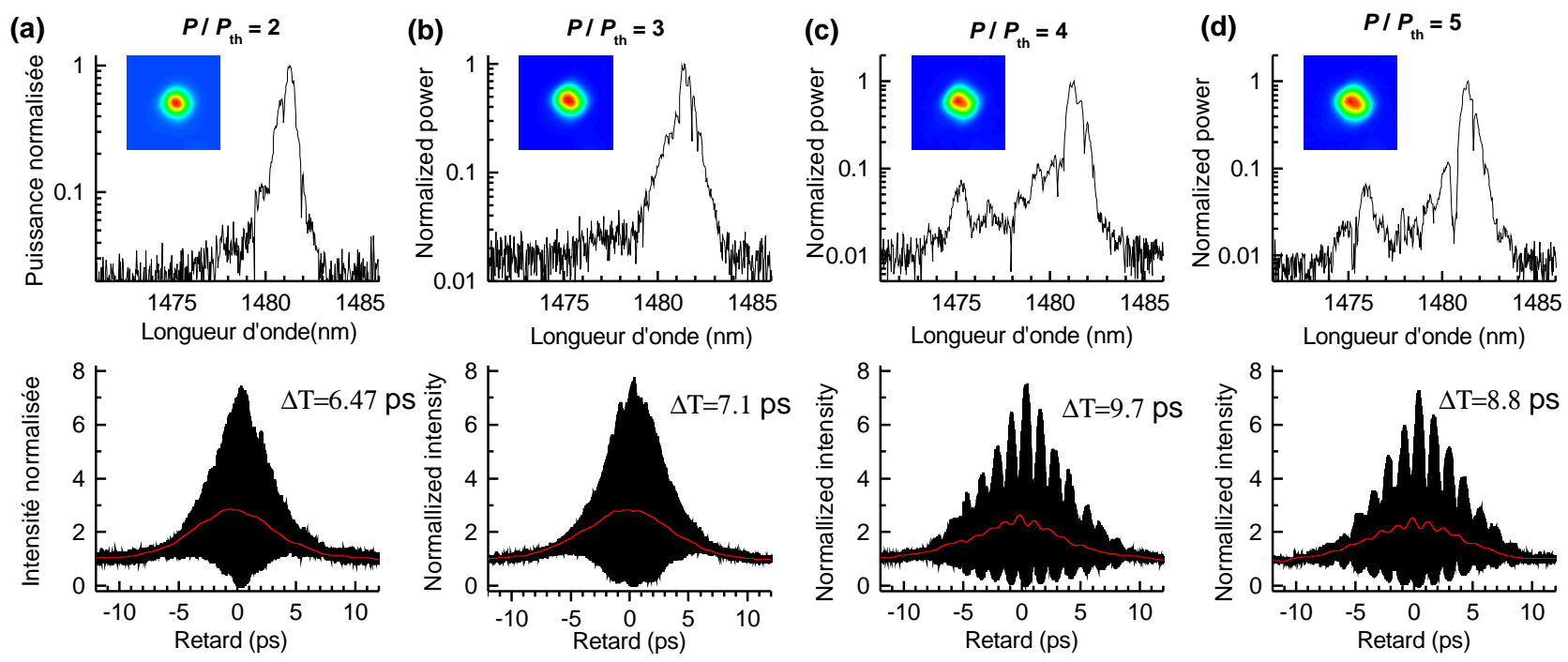

Fig. 10. Experimental signal spectrum, beam profile and autocorrelation trace for several pumping rates for QPM grating G4 with a positive QPM chirp rate, a $150 \mu \mathrm{m}$ pump waist, and $R_{\mathrm{oc}}=90 \%$.

peak. A red side-band is generated right after the threshold and continues to grow as the pump power increases, while a blue side-band begins to be generated only at high pump power.

\section{B. Numerical investigation}

Numerical simulations were carried out to further investigate the spectral properties. First, we studied the evolution of the parametric waves inside the chirped QPM crystal while the $\mathrm{OPO}$ is in steady-state regime (after a sufficient number of cavity round-trips to have identical spectrum and pulse shapes from one round-trip to the other). For simplicity's sake, self-phase modulation (SPM) has been discarded in the numerical modeling since it as a marginal effect in our case. Indeed, the involved intra-cavity intensity remains below $300 \mathrm{MW} / \mathrm{cm}^{2}$, leading to an estimated nonlinear phase due to SPM smaller than $0.1 \pi$ for a nonlinear index coefficient $n_{2}=50 \times 10^{-20} \mathrm{~m}^{2} / \mathrm{W}$, among the highest reported values for $\mathrm{LiNbO}_{3}$ [20].

As shown in Fig. 12(a) and (b) for grating G6', the calculated steady-state signal spectrum at the output of the APPLN crystal has an asymmetrical shape, consisting of a main lobe with smaller side-lobes on one side, which is in agreement with the experimental results. The effect of the chirp rate sign is also consistent with the experimental results concerning the location of the side-lobes on either the red or the blue side of the main peak. However, one should also notice that a change of the chirp rate sign results in a mirror symmetry of the calculated spectrum and that the numerical simulation does not recover the irregular spectra that are experimentally observed for positive chirps. The origin of this discrepancy still needs to be determined. We can nevertheless assume that it is due to phenomena that are not taken into account by our model. Since it only considers plane waves, transverse effects or non-collinear interactions, particular to chirped QPM crystal, could induce this kind of dependence on the chirp sign [21].

To gain some physical insights, we also studied the evolution of the signal spectrum inside the crystal from which the incident 


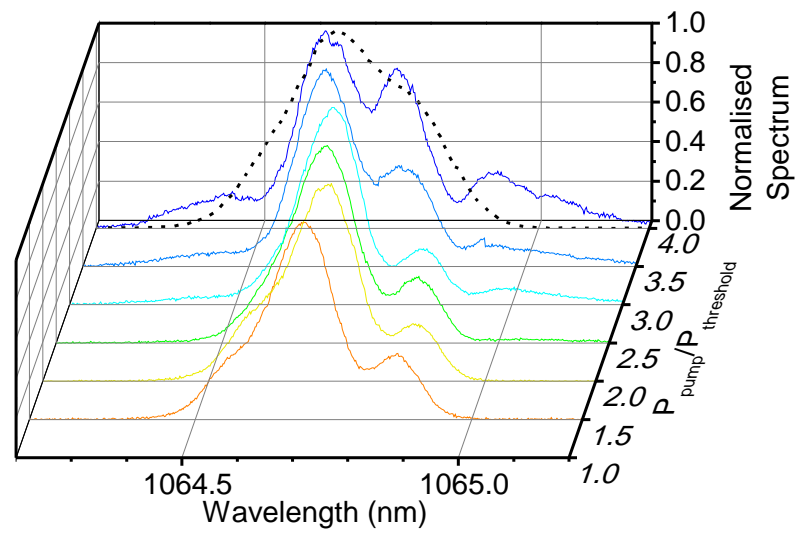

Fig. 11. Experimental pump spectrum after passing through the APPLN crystal (after mirror M3 in Fig. 4) for several pumping rates $P_{\text {pump }} / P_{\text {threshold }}$ for QPM grating G6' with a negative chirp rate, a pump waist radius of $110 \mu \mathrm{m}$, and $R_{\mathrm{OC}}=98 \%$ (Solid lines: the OPO is oscillating; black dashed line: the OPO is intentionally misaligned to avoid oscillation and the pump power is equivalent to a pumping rate of 4 times the oscillation threshold).

signal has been subtracted, in other words the increment of signal due to parametric amplification inside the crystal, as shown in Fig. 12(c) and (d), for respectively a negative and a positive chirp rate. As these two figures are basically symmetric with respect to each another, we can limit the following study to the case of the negative chirp rate without loss of generality.

To help the analysis, we plot in Fig. 13(c) the signal increment for discrete signal wavelengths labeled $\mathrm{s}_{-1}, \mathrm{~s}_{0}, \mathrm{~s}_{1}$, and $\mathrm{s}_{2}$ whose values are shown on the signal spectrum in Fig. 13(a). Wavelength $\mathrm{s}_{0}$ corresponds to the main peak of the signal spectum while wavelength $s_{2}$ corresponds to the first valley. Wavelengths $s_{-1}$ and $s_{1}$ are located at mid-peak on both side of wavelength $s_{0}$. Let us first underline that, since the OPO is in steady-state, the overall parametric gain experienced for all these signal wavelengths is the same and is exactly equal to the round-trip loss. Here, we discuss how this gain is distributed along the propagation in the nonlinear crystal.

We notice that the signal increment at wavelength $\mathrm{s}_{0}$ exhibits a similar growth as the one expected for a monochromatic wave in a chirped QPM crystal, as reported in previous works [1, 18], with sharp amplification around the perfect quasiphase matching point (PPMP) at $z_{\mathrm{PPMP}}$, which is the point where $\Delta k\left(z_{\text {PPMP }}\right)=k_{\mathrm{p}}-k_{\mathrm{s}}-k_{\mathrm{i}}-K_{\mathrm{g}}\left(z_{\mathrm{PPMP}}\right)=0$. This sharp amplification is then followed by a damped oscillatory behavior, as expected for an apodized QPM grating. The valley wavelength $\mathrm{s}_{2}$, and the mid-peak wavelength $\mathrm{s}_{1}$, present a similar initial growth around the PPMP, however a decreasing oscillatory behavior is then observed along the remaining of the crystal. The mid-peak $\mathrm{s}_{-1}$ evolution is distinct since it starts by a decrease at the beginning of the crystal, followed by a sharp growth around the PPMP, and then a slower growth with a damped oscillatory behavior over the rest of the crystal.

To investigate the link between the pump and the signal sidelobes, we also plot the pump spectrum and its evolution during propagation in the nonlinear crystal for both chirp signs (see Fig. 14 ). In this figure, the top subplots (a) and (b) represent the input and output pump spectra. One can observe that the pump spectrum is reshaped during propagation through the
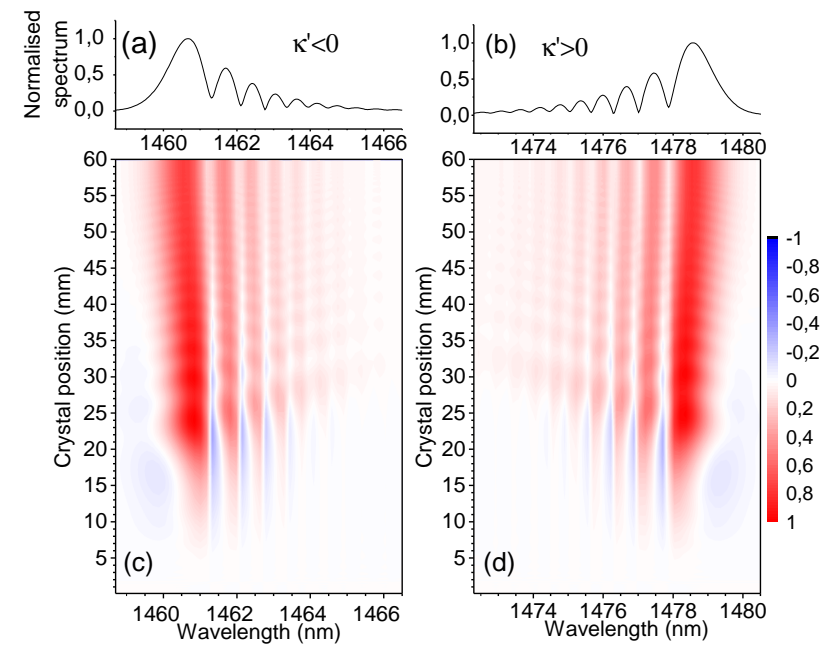

Fig. 12. Calculated normalized steady-state signal spectrum at crystal output, for grating $\mathrm{G6}^{\prime}$, for negative (a) and positive QPM chirp rate (b), and corresponding spectrograms of the evolution of the signal spectrum, from which the incident signal spectrum has been subtracted, along the propagation of the pulse in the crystal, for a negative (c) and a positive QPM chirp (d).
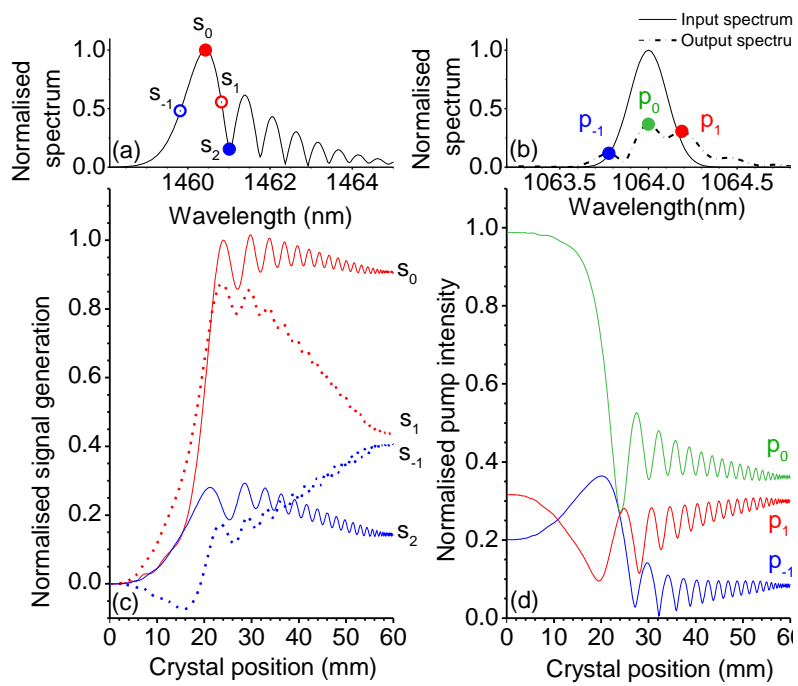

Fig. 13. Calculated normalized output signal spectrum (a) and incident and output pump spectra (b). Normalized signal generation (signal intensity from which the initial value at $z=0$ has been subtracted) at discrete signal (c) and pump (d) wavelengths as function of the position in the nonlinear crystal, for a pumping rate of 4 times the oscillation threshold. The discrete signal and pump wavelengths in (c) and (d) are indicated in their respective output spectrum in (a) and (b).

crystal with the emergence of side-bands while the central peak is depleted, which is in agreement with the experimental results shown in Fig. 11. We note that, as in Fig. 12, the difference between the two chirp signs results in a simple symmetry, which means that only one chirp sign can be studied. We also plot in Fig. 13 (b) and (d) the evolution of particular discrete pump wavelengths, for a negative QPM chirp rate. In this figure, wavelength $\mathrm{p}_{0}$ is the central wavelength of the incident Gaussian 


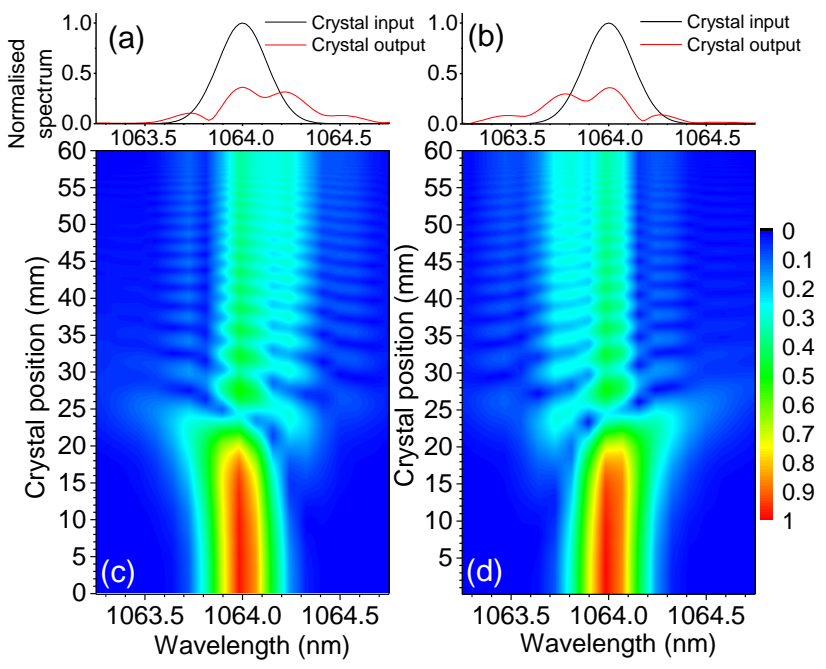

Fig. 14. Normalized incident pump spectrum (black) and calculated steady-state pump spectrum at crystal output (red), for grating $\mathrm{G6}^{\prime}$, for a negative (a) and a positive QPM chirp rate (b), and corresponding spectrograms of the evolution of the pump spectrum along the propagation of the pulse in the crystal, for a negative (c) and a positive QPM chirp rate (d).

pump spectrum; $\mathrm{p}_{-1}$ and $\mathrm{p}_{1}$ correspond to the peak value of the two side-bands generated on each side of $\mathrm{p}_{0}$. One can notice that $\mathrm{p}_{0}$ exhibits a similar depletion as reported in previous work devoted to frequency conversion of monochromatic waves in chirped QPM nonlinear media with a sharp decrease at the same position $(z \simeq 20 \mathrm{~mm}$ ) as signal increment exhibits a sharp increase, followed by a damped oscillatory behavior. The red side-band $\mathrm{p}_{1}$ starts being depleted before $\mathrm{p}_{0}$, it then reaches a minimum at the same position as $\mathrm{p}_{0}$ experiences a sharp depletion. After this minimum, $\mathrm{p}_{1}$ exhibits a slow growth with a damped oscillatory behavior around the increasing mean value. In contrast, the blue side-band $\mathrm{p}_{-1}$ first increases in the crystal beginning, while the other two wavelengths $\mathrm{p}_{0}$ and $\mathrm{p}_{1}$ decrease. It then reaches a maximum at a position that is close to the minimum for $\mathrm{p}_{1}$ before experiencing a sharp decrease, and lastly a damped oscillatory behavior. At the crystal output, the intensity at wavelength $p_{-1}$ is eventually much smaller that for $p_{0}$ and $\mathrm{p}_{1}$.

\section{Discussion on the origin of spectral modulations}

These atypical signal spectrum modulations are particular to picosecond OPO based on an aperiodic QPM crystal, and have never, to the best of our knowledge, been reported or explained before.

Since the QPM grating is chirped, the different wavelengths of the signal spectrum are not quasi-phase matched for DFG with the pump central wavelength $\mathrm{p}_{0}$ at the same position, i.e. PPMP position. For a negative chirp rate, the PPMP first occurs for short wavelengths while it is the opposite for positive chirp rate. The main peak of the signal spectrum is thus phase matched before the other side-lobes during propagation through the nonlinear crystal for both chirp rate signs. As a consequence, the pump pulse first interacts with the main signal peak and experiences depletion. The available pump power for parametric amplification of the following side-lobes is then gradually lowered along propagation in the nonlinear crystal. To maintain a constant amplification factor over the whole signal spectrum, the height of

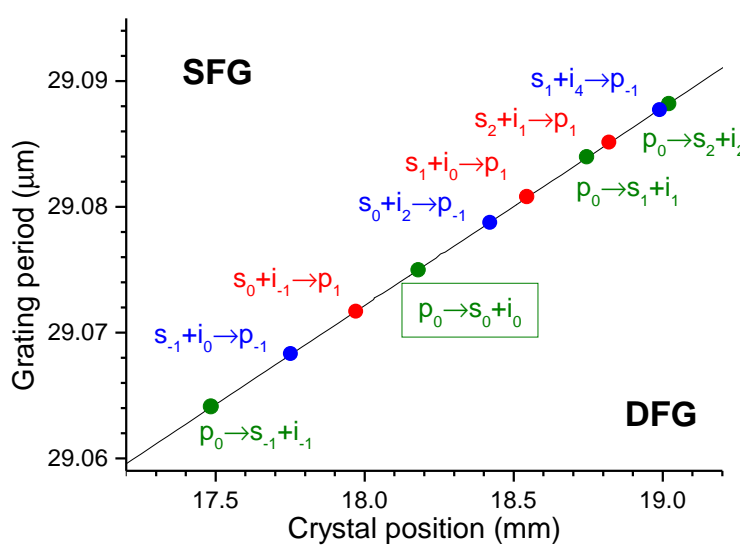

Fig. 15. Position of the perfect phase matching point of the different parametric processes in the nonlinear crystal for grating G6', for the DFG process (in green) with a $1064 \mathrm{~nm}$ pump wavelength, and the generation of the red and blue pump sideband via SFG. The subscripts refer to the discrete wavelengths defined in Fig. 13.

the successive side-lobes must thus be smaller and smaller. This position-dependence of the signal wavelength which is phasematched for DFG with $\lambda_{\mathrm{p}_{0}}$ is particular to chirped QPM and can explain the asymmetrical shape of the overall signal spectrum envelope. However, it does not explain the presence of modulation within the spectrum envelope, resulting in the observed signal spectrum consisting of well separated side-lobes.

To understand the origin of this signal modulation, we have to study the relation between the signal side-lobes, and the pump side-bands. Indeed, by comparing Fig. 9 and Fig. 11, one can see that the emergence of the pump side-bands is associated with the formation of the signal spectral modulation.

To generate a pump side-band, sum frequency generation (SFG) must occur between signal and idler wavelengths such as $1 / \lambda_{\mathrm{s}}+1 / \lambda_{\mathrm{i}} \neq 1 / \lambda_{\mathrm{p}_{0}}$, which can be referred as cross interaction. For instance, to generate a blue pump side-band $\left(\lambda_{\mathrm{p}}<\lambda_{\mathrm{p}_{0}}\right)$, $\lambda_{\mathrm{s}_{0}}$ must be mixed with a wavelength $\lambda_{\mathrm{i}}$ that is shorter than $\lambda_{\mathrm{i}_{0}}$, where $1 / \lambda_{\mathrm{s}_{0}}+1 / \lambda_{\mathrm{i}_{0}}=1 / \lambda_{\mathrm{p}_{0}}$. To generate a red pump side-band $\left(\lambda_{\mathrm{p}}>\lambda_{\mathrm{p}_{0}}\right)$, we need $\lambda_{\mathrm{i}}>\lambda_{\mathrm{i}_{0}}$. To explain the observed spectral features, one has thus to take into account all the present wavelengths, with all the resulting possible parametric interactions.

To analyze the effect of these cross interactions on the modulation of the signal spectrum, it is useful to observe the variation of discrete wavelengths as represented in Fig. 13(c) and (d). As already discussed, the intensities of the main peaks of the pump $\left(\mathrm{p}_{0}\right)$ and signal $\left(\mathrm{s}_{0}\right)$ evolve in the crystal very similarly to monochromatic waves: there is a sharp energy transfer between pump and signal around the PPMP (from approximately 15 to $24 \mathrm{~mm}$ inside the crystal), followed by damped oscillation [1]. The signal minimum $s_{2}$ also increases sharply when the main pump peak $\mathrm{p}_{0}$ is depleted. However, its decreasing oscillatory behavior seems more correlated with the growth of the pump blue side-band $\mathrm{p}_{-1}$ near the end of the crystal.

The other wavelengths $s_{1}$ and $s_{-1}$ display more subtle intensity evolution inside the crystal. The early increase of $s_{1}$ wavelength, and its following decreasing oscillatory behavior at the end of the crystal, seems to be linked with the early depletion of $\mathrm{p}_{1}$, and its increasing oscillatory behavior near crystal output. 


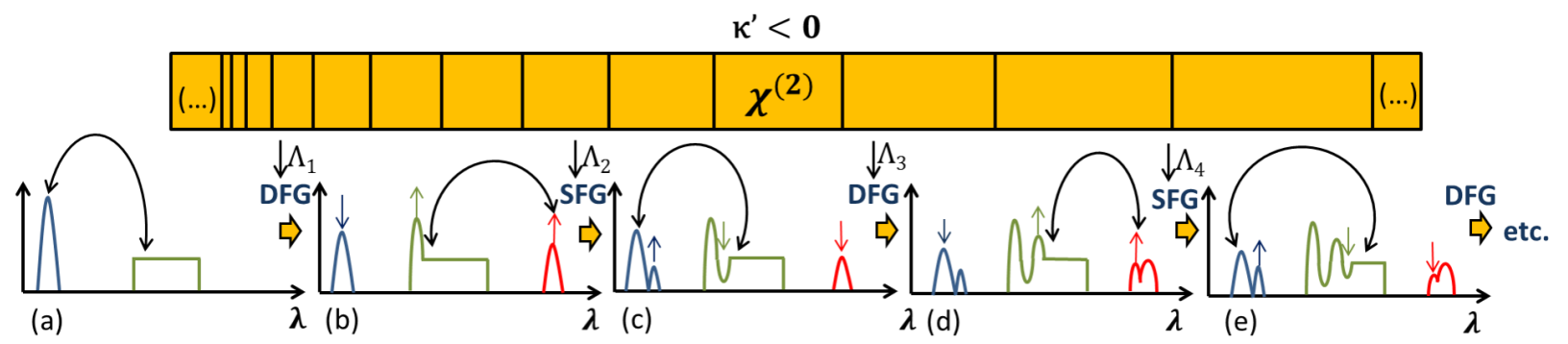

Fig. 16. Schematic diagram of the generation of the signal spectrum modulation and of the pump red side-band, in an aperiodic QPM crystal (with $\kappa^{\prime}<0$ ), through cascaded DFG/SFG processes. The blue, green and red profiles respectively correspond to the pump, signal and idler spectra. From (a) to (e) the spectra are presented for different QPM grating periods, and the likely parametric interaction for each period (black arrow).

Wavelength $s_{-1}$ is the only signal wavelength which decreases at crystal beginning, before experiencing an increasing oscillatory behavior. Its behavior seems to be linked with the early increase of $p_{-1}$ which is then followed by its depletion. From an analysis of these particular wavelengths, we can see that several cross interactions are expected to occur between different parts of the signal, pump, and idler spectra. Indeed, each wavelength can experience several parametric processes that may be at the origin of the observed modulation of the signal spectrum.

Another point to take into consideration is the position of the perfect phase matching point (PPMP) for each process. Fig. 15 gives the PPMP position of the different parametric processes involving the discrete wavelengths defined in Fig. 13. The OPO cavity being singly resonant at the signal, there is no idler at the crystal input. As a consequence, to generate a pump sideband by a SFG process, the required idler wavelength has to be first generated via a DFG process. As seen in Fig. 13, this condition is satisfied for all the SFGs that generate the pump red side-band (red point in the figure), as each corresponding PPMP is situated after the necessary DFG process (green point in the figure), e.g., the green point $\mathrm{p}_{0} \rightarrow \mathrm{s}_{-1}+\mathrm{i}_{-1}$ is before the red point $\mathrm{s}_{0}+\mathrm{i}_{-1} \rightarrow \mathrm{p}_{1}$. For the SFG process at the origin of the blue side-band, its PPMP is situated before the DFG PPMP, which means that to explain its generation, one has to take into account that the wave mixing processes take places over finite (i.e., non-zero) parametric interaction lengths and not only at the PPMP position.

Yaakobi et al. developed a general theory of autoresonnant three-waves mixing in nonuniform $\chi^{(2)}$ media [18]. From the coupled wave equations and the use of a few approximations, they derived an analytical solution of the waves evolution in such media. This solution is valid if the power of one of the parametric waves is much higher than that of the two other waves. Even through their solution is not strictly valid in our OPO, we can nevertheless extract from their work general trends. The interaction length of a parametric process is slowly dependent on the wavelength and refractive index, inversely dependent on the chirp rate $\kappa^{\prime}$, and linearly dependent to the input signal and pump electrical field amplitude. The finite value of the interaction length, which can extend to a few millimeters at high power, can explain why the generation of the blue pump side-band by SFG: $\mathrm{s}_{-1}+\mathrm{i}_{0} \rightarrow \mathrm{p}_{-1}$ is possible, while its PPMP is situated before the PPMP for the necessary idler generation by DFG: $\mathrm{p}_{0} \rightarrow \mathrm{s}_{0}+\mathrm{i}_{0}$. As blue side-band generation is a phenomenon which occurs at high pump power, while the signal spectral side lobes generation already starts to develop at low power, we can conclude that the blue side-band generation is mainly a second-order side effect which is a consequence of the signal modulations but only plays a very marginal role in their generation.

We can now propose the explanation for the generation of the signal side-lobes which is depicted in Fig. 16. As discussed above, the pump blue side-band can be discarded since it plays only a marginal role. As shown in Fig 16(a), since the OPO is singly resonant, only the pump and signal spectra are present at the crystal entrance. Moreover, for simplicity's sake, we consider here that the incident signal has a top-hat profile, and schematize the process from the $\mathrm{p}_{0} \rightarrow \mathrm{s}_{0}+\mathrm{i}_{0}$ interaction of Fig. 15. Then, the pulses propagate to the PPMP for DFG between the incident pump and the short-wavelength side of the signal spectrum, corresponding to grating period $\Lambda_{1}$. This first DFG process leads to the depletion of the pump and the amplification of the signal, resulting in the development of the main signal lobe and the corresponding idler lobe, as depicted in Fig 16(b). This basically corresponds to the DFG process: $\mathrm{p}_{0} \rightarrow \mathrm{s}_{0}+\mathrm{i}_{0}$. After further propagation, the pulses cross grating period $\Lambda_{2}$ representing the PPMP for SFG process: $i_{0}+s_{1} \rightarrow p_{1}$. As shown in Fig 16(c), this leads to the depletion of the idler lobe, the development of the first signal valley and the emergence of the pump red side-band. Further away, PPMP occurs for a new DFG process for a longer signal wavelength, $\lambda_{\mathrm{s}_{4}}$, at grating period $\Lambda_{3}$. As depicted in Fig 16(d), this induces the development of the second signal and idler lobes of lower peak power due to the lower available pump peak power. Then, as shown in Fig 16(e), the second idler lobe and signal valley are phase-matched for grating period $\Lambda_{4}$ where the SFG process leads to a new enhancement of the pump red side-band. This cascaded DFG/SFG interaction then continues until the main pump peak is too low to provide further parametric gain.

Actually, the involved interactions are more complex than in this simplified picture. Indeed, due to their finite interaction lengths, several SFG and DFG processes can coexist. For instance, while the SFG with the first signal valley $\left(\mathrm{i}_{1}+\mathrm{s}_{2} \rightarrow \mathrm{p}_{1}\right)$ leads to the development of the pump red side-band, this same signal wavelength is also amplified by the DFG process with the pump main peak $\left(\mathrm{p}_{0} \rightarrow \mathrm{s}_{2}+\mathrm{i}_{2}\right)$. The coexistence of these two processes involving the same signal wavelength $\lambda_{\mathrm{s}_{2}}$ yields a weaker idler generation and thus to a minimum in the idler spectrum. The subsequent SFG process: $i_{2}+s_{4} \rightarrow p_{1}$, involving this idler wavelength with the next signal peak is hence weakened, leading to a dominant DFG process: $\mathrm{p}_{0} \rightarrow \mathrm{s}_{4}+\mathrm{i}_{4}$, which can explain the generation of the second signal peak. The occur- 


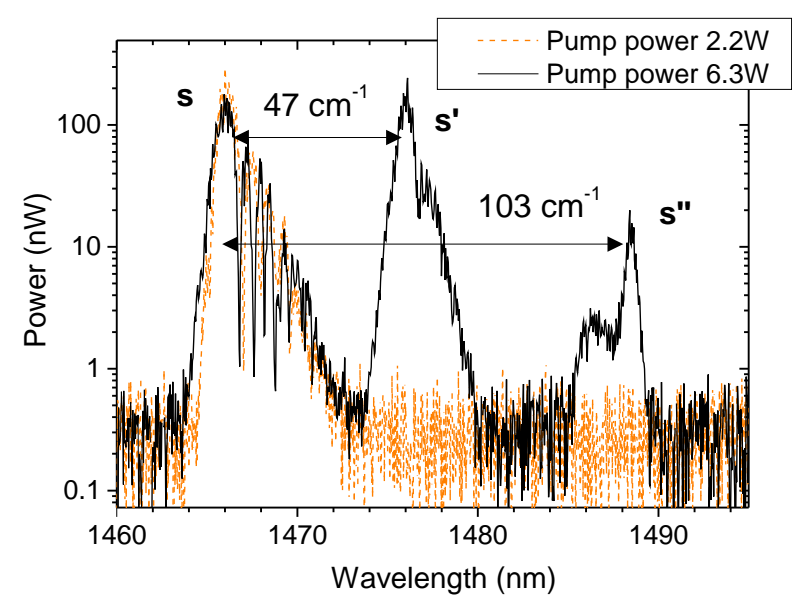

Fig. 17. OPO output spectrum in signal wavelength range with grating $\mathrm{G6}^{\prime}$, for a negative QPM chirp rate, for low $(2.2 \mathrm{~W})$ and high $(6.3 \mathrm{~W})$ incident pump power. The spectral feature labeled $s$ corresponds to the expected OPO signal spectrum. For a pump power of $2.7 \mathrm{~W}$, a secondary peak, labeled s', appears with a shift of $47 \mathrm{~cm}^{-1}$. Above an input pump power of $3.8 \mathrm{~W}$ a third peak, labeled s", appears with a shift of 103 $\mathrm{cm}^{-1}$.

rences of either peaks or valleys in the signal spectrum is thus related to which parametric process is dominating between DFG and SFG. This dominance depends on the available generated idler power, which itself depends on the previous prevailing parametric process earlier in the crystal.

We can thus assume that it is the cascaded DFG/SFG processes-phase-matched at different successive positions, due to the aperiodic QPM crystal-that are at the origin of the signal spectral modulation and the pump side-band generation. The distance between two peaks depends on the interaction length of the different parametric processes. This interaction length is typically of a few millimeters, depending on the incident pump peak intensity and inversely proportional to the grating chirp.

\section{TERAHERTZ CASCADING EFFECT AT HIGH PUMP POWER}

As the pump becomes more powerful, the signal spectrum is further altered, with the emergence of two additional secondary peaks. These additional peaks are well separated from the initial signal spectrum and appear at a spectral distance which is typically ten times larger than the distance between the previously investigated side lobes.

Fig. 17 represents an example of this phenomenon, for G6' QPM grating with a negative chirp rate. The initial signal spectrum corresponds to the spectral feature labeled s in Fig. 17. One could notice that a spectral difference of $+5 \mathrm{~nm}$ is observed when compared to numerical simulation shown in Fig. 12. This difference may be due to the focusing of the pump beam in the crystal center, which is not taken into account by the plane-wave calculation, leading to a spatial shift of the QPM grating position where the first signal lobe develops.

The first secondary signal peak (labelled s' in Fig. 17) is located at a spectral spacing of about $10.2 \mathrm{~nm}\left(47 \mathrm{~cm}^{-1}\right)$ from the main peak while the second secondary signal peak, $\mathrm{s}^{\prime \prime}$, is located at approximately $22.2 \mathrm{~nm}\left(103 \mathrm{~cm}^{-1}\right)$. They respectively appear for pump powers of approximately two and three times

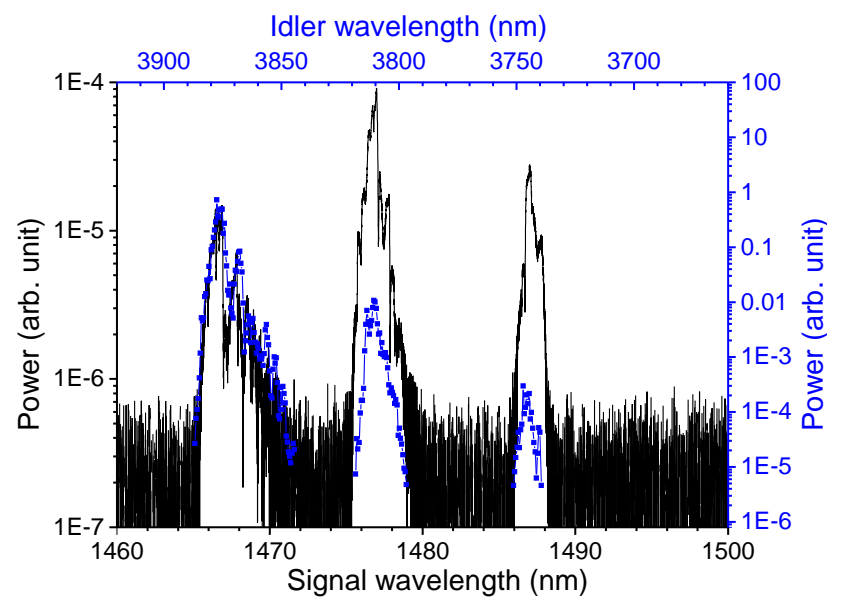

Fig. 18. OPO output spectra in the near-infrared in signal wavelength range (black) and in the mid-infrared in idler wavelength range (blue).

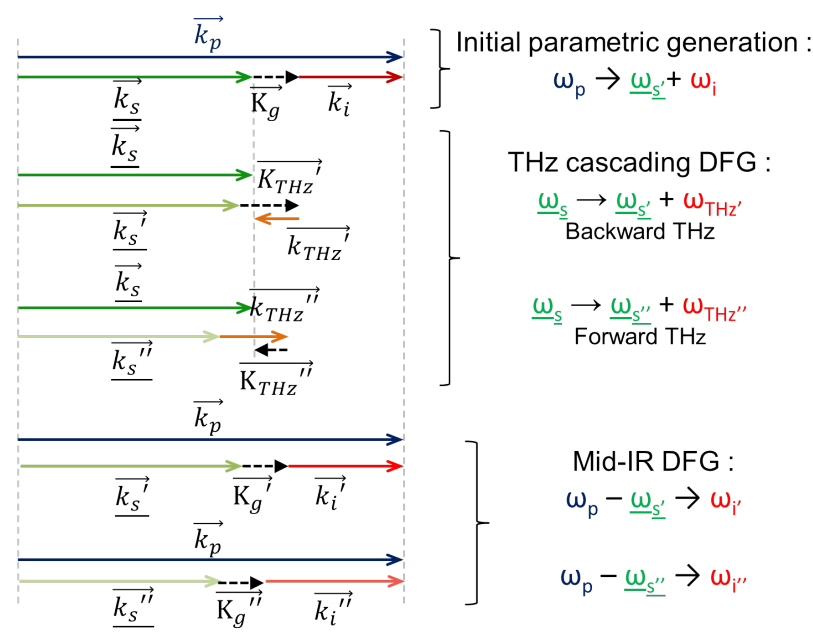

Fig. 19. Schemes of the various wave-vector conservation conditions for the initial parametric interaction, the terahertz cascading parametric processes, and the mid-infrared parasitic difference frequency generation. Underlined frequency are resonant in the OPO cavity.

above the oscillation threshold. These peaks can appear outside the parametric gain bandwidth of the APPLN crystal, for each grating and each sign of the chirp rate. As their polarization is not perpendicular to the main peak polarization, they are not expected to be related to any Raman shift. Indeed, the first referred Raman peak with extraordinary-wave polarization occurs at a spectral shilft around $255 \mathrm{~cm}^{-1}$ [22-24].

As previously reported by Kiessling et al. [25] in a continuouswave PPLN OPO, and later by Liu et al. [26] with a picosecond OPO based on a MgO-doped PPLN crystal, we expect that these secondary peaks are due to cascaded OPO operation, pumped by the circulating signal pulses, delivering an idler radiation in the terahertz spectral range. As the primary signal s grows inside the cavity, it can act as a pump of two new resonant secondary signal waves $\mathrm{s}^{\prime}$ and $\mathrm{s}^{\prime \prime}$.

Even though the direct observation of terahertz waves is not possible with our experimental setup, we can still analyze the emitted spectrum in the mid-infrared range, around the 
primary idler wavelength, with the use of a monochromator (iHR320 - Horiba). In Fig. 18, we overlap the spectra of the signal (obtained with the OSA) and of the idler (obtained with the monochromator). The horizontal axes for signal and idler are linked by the energy conservation with a pump at $1064 \mathrm{~nm}$. For each signal peak, we can find the paired idler peak in the mid-infrared. Whereas all the signal peaks have comparable powers, the power ratio between the main idler peak and the two others peaks is at least $20 \mathrm{~dB}$. This indicates that secondary signal peaks $\mathrm{s}^{\prime}$ and $\mathrm{s}^{\prime \prime}$ are not generated by parametric oscillation pumped at $1064 \mathrm{~nm}$. Indeed, the relatively low magnitude of the two secondary peaks at $3.75 \mu \mathrm{m}$ and $3.8 \mu \mathrm{m}$, close to the idler peak at $3.87 \mu \mathrm{m}$, indicates that they are rather generated by parasitic DFG between the pump at $1064 \mathrm{~nm}$ and the signal $\mathrm{s}^{\prime}$ and $\mathrm{s}^{\prime \prime}$ which are themselves generated by cascading OPO process. Moreover, as detailed in Sec. 5, the 1064-nm pump pulse is depleted by the primary signal parametric amplification and has a too low peak intensity after the $z$ position corresponding to the PPMP of the weakest side-lobe to be the pump of $s^{\prime}$ and $\mathrm{s}^{\prime \prime}$ further away in the crystal. These findings thus support the occurrence of cascaded terahertz OPO interaction.

Fig. 19 summaries the various parametric processes which occur in the optical cavity by a representation of the different wave-vector diagrams. The subscripts $p, s$ and i represent respectively pump, signal and idler, while the subscript $\mathrm{THz}$ corresponds to the idler terahertz waves generated by the cascading OPO process. The underlined elements designate the waves resonating in the OPO. To satisfy the QPM condition, the terahertz wave can be either counter-propagative (when associated with signal $\mathrm{s}^{\prime}$ ) or co-propagative (when associated with signal $\mathrm{s}^{\prime \prime}$ ), corresponding respectively to backward and forward terahertz waves. In the investigated configuration, the forward terahertz parametric generation is quasi-phase-matched for the shortest periods of the crystal, while the backward terahertz is generated for the longest periods [27]. As seen in Fig. 18, the magnitude of cascading signal $\mathrm{s}^{\prime}$ can be several $\mathrm{dB}$ higher than the one of initial signal $s$. This suggests that an APPLN crystal could be a relevant nonlinear medium for terahertz-wave generation.

\section{CONCLUSION}

An experimental and numerical investigation of the properties of a broadband picosecond synchronously-pumped optical parametric oscillator based on a chirped quasi-phase matched crystal has been reported. Compared to a crystal with a periodic QPM grating, this chirped QPM crystal gives a wide parametric gain spectrum which can extend to $275 \mathrm{~nm}$ in the mid-infrared in our case. Despite of the parametric gain reduction due to this broadening, a conversion efficiency up to $33 \%$ has been obtained. Moreover, with an 60-mm-long APPLN crystal providing the same oscillation threshold as a 20-mm-long PPLN, we observe a strong reduction of saturation effects at high pump power.

We also have carried out a detailed study of the particular spectral properties of such a picosecond SPOPO based on chirped QPM. We have shown that the generated signal spectrum consists of a main peak with several lower-magnitude side-lobes on one side. This signal spectral modulation is dependent on the incident pump power, pulse duration and on the QPM chirp rate (magnitude and sign). These previously unreported spectral features have been modeled and interpreted in terms of cascaded DFG/SFG processes which take place at different positions in the nonlinear medium. Indeed, owing to the aperiodic grating, perfect phase matching points exist for each possible parametric process. Comparable spectral features are obtained at other OPO wavelengths for other central QPM grating periods or temperatures.

At high pump power, cascading parametric oscillationpumped by the intracavity signal pulses-has also been observed, leading to the emergence of additional secondary signal peaks shifted by $10.2 \mathrm{~nm}$ and $22.2 \mathrm{~nm}$ from the original signal spectrum. The corresponding idler radiations of these cascading parametric conversions are expected to be terahertz-waves.

Acknowledgment. The authors acknowledge Cédric Laporte for his help on the experiments. We are also grateful to Prof. Derryck T. Reid for helpful discussions on the OPO numerical simulation.

Funding. Agence Nationale de la Recherche (ANR) (ANR-14CE26-0036); "Investissements d'Avenir" LabEx PALM (SYCLOP and SORA) (ANR-10-LABX-0039).

Disclosures. The authors declare that there are no conflicts of interest related to this article.

\section{REFERENCES}

1. M. Charbonneau-Lefort, B. Afeyan, and M. M. Fejer, "Optical parametric amplifiers using chirped quasi-phase-matching gratings I: practical design formulas," J. Opt. Soc. Am. B 25, 463-480 (2008).

2. G. M. Gale, F. Hache, and M. Cavallari, "Broad-bandwidth parametric amplification in the visible: femtosecond experiments and simulations," IEEE J. Sel. Top. Quantum Electron. 4, 224-229 (1998).

3. M. Yamada, N. Nada, M. Saitoh, and K. Watanabe, "First-order quasiphase matched $\mathrm{LiNbO}_{3}$ waveguide periodically poled by applying an external field for efficient blue second-harmonic generation," Appl. Phys. Lett. 62, 435-436 (1993).

4. C. R. Phillips, B. W. Mayer, L. Gallmann, M. M. Fejer, and U. Keller, "Design constraints of optical parametric chirped pulse amplification based on chirped quasi-phase-matching gratings," Opt. Express 22, 9627-9658 (2014).

5. K. A. Tillman, D. T. Reid, D. Artigas, J. Hellström, V. Pasiskevicius, and F. Laurell, "Low-threshold femtosecond optical parametric oscillator based on chirped-pulse frequency conversion," Opt. Lett. 28, 543-545 (2003).

6. D. Artigas and D. T. Reid, "Efficient femtosecond optical parametric oscillators based on aperiodically poled nonlinear crystals," Opt. Lett. 27, 851-853 (2002).

7. K. A. Tillman and D. T. Reid, "Monolithic optical parametric oscillator using chirped quasi-phase matching," Opt. Lett. 32, 1548-1550 (2007).

8. T. W. Neely, L. Nugent-Glandorf, F. Adler, and S. A. Diddams, "Broadband mid-infrared frequency upconversion and spectroscopy with an aperiodically poled $\mathrm{LiNbO}_{3}$ waveguide," Opt. Lett. 37, 4332-4334 (2012).

9. D. Descloux, J.-B. Dherbecourt, J.-M. Melkonian, M. Raybaut, J.-Y. Lai, C. Drag, and A. Godard, "Rapidly tunable optical parametric oscillator based on aperiodic quasi-phase matching," Opt. Express 24, 1111211125 (2016).

10. D. Descloux, G. Walter, E. Cadiou, J.-B. Dherbecourt, G. Gorju, J.M. Melkonian, M. Raybaut, C. Drag, and A. Godard, "Wide and fast dispersion tuning of a picosecond OPO based on aperiodic quasiphase matching using an axially chirped volume Bragg grating," Opt. Lett. 41, 4060-4063 (2016).

11. D. Descloux, C. Laporte, J. B. Dherbecourt, J. M. Melkonian, M. Raybaut, C. Drag, and A. Godard, "Spectrotemporal dynamics of a picosecond OPO based on chirped quasi-phase-matching," Opt. Lett. 40, 280-283 (2015).

12. D. Descloux, C. Laporte, J.-B. Dherbecourt, J.-M. Melkonian, M. Raybaut, C. Drag, and A. Godard, "Spectrotemporal dynamics of a picosecond OPO based on chirped quasi-phase-matching: erratum," Opt. Lett. 43, 494-494 (2018).

13. C. Laporte, J.-B. Dherbecourt, J.-M. Melkonian, M. Raybaut, C. Drag, and A. Godard, "Analysis of cavity-length detuning in diffraction-grating 
narrowed picosecond optical parametric oscillators," J. Opt. Soc. Am. B 31, 1026-1034 (2014).

14. O. Gayer, Z. Sacks, E. Galun, and A. Arie, "Temperature and wavelength dependent refractive index equations for $\mathrm{MgO}$-doped congruent and stoichiometric $\mathrm{LiNbO}_{3}$," Appl. Phys. B 91, 343-348 (2008).

15. G. Arisholm, "General analysis of group velocity effects in collinear optical parametric amplifiers and generators," Opt. Express 15, 65136527 (2007).

16. D. C. Hanna, M. V. O'Connor, M. a. Watson, and D. P. Shepherd, "Synchronously pumped optical parametric oscillator with diffractiongrating tuning," J. Phys. D 34, 2440-2454 (2001).

17. H. Suchowski, V. Prabhudesai, D. Oron, A. Arie, and Y. Silberberg, "Robust adiabatic sum frequency conversion," Opt. Express 17, 1273112740 (2009).

18. O. Yaakobi, L. Caspani, M. Clerici, F. Vidal, and R. Morandotti, "Complete energy conversion by autoresonant three-wave mixing in nonuniform media," Opt. Express 21, 1623-1632 (2013).

19. C. R. Phillips, C. Langrock, D. Chang, Y. W. Lin, L. Gallmann, and M. M. Fejer, "Apodization of chirped quasi-phasematching devices," J. Opt. Soc. Am. B 30, 1551-1568 (2013).

20. M. Bache and F. W. Wise, "Type-I cascaded quadratic soliton compression in lithium niobate: Compressing femtosecond pulses from high-power fiber lasers," Phys. Rev. A 81, 053815 (2010).

21. M. Charbonneau-Lefort, B. Afeyan, and M. M. Fejer, "Competing collinear and noncollinear interactions in chirped quasi-phase-matched optical parametric amplifiers," J. Opt. Soc. Am. B 25, 1402-1413 (2008).

22. R. W. Boyd, Nonlinear Optics, Third Edition (Academic Press, 2008).

23. N. V. Surovtsev, V. K. Malinovskii, A. M. Pugachev, and A. P. Shebanin, "The nature of low-frequency raman scattering in congruent melting crystals of lithium niobate," Phys. Solid State 45, 534-541 (2003).

24. C. R. Phillips, C. Langrock, J. S. Pelc, M. M. Fejer, I. Hartl, and M. E. Fermann, "Supercontinuum generation in quasi-phasematched waveguides," Opt. Express 19, 18754-18773 (2011).

25. J. Kiessling, R. Sowade, I. Breunig, K. Buse, and V. Dierolf, "Cascaded optical parametric oscillations generating tunable terahertz waves in periodically poled lithium niobate crystals," Opt. Express 17, 87-91 (2009).

26. T. Liu, L. Liu, X. B. Wang, X. Li, and J. Hou, "Cascaded synchronous terahertz optical parametric oscillations in a single MgO:PPLN crystal"," Laser Phys. 22, 678-683 (2012).

27. J. Kiessling, K. Buse, and I. Breunig, "Temperature-dependent sellmeier equation for the extraordinary refractive index of $5 \mathrm{~mol}$. \% MgOdoped $\mathrm{LiNbO}_{3}$ in the terahertz range," J. Opt. Soc. Am. B 30, 950-952 (2013). 